\title{
High Throughput Grain Mapping with Sub-Nanometer Resolution by 4D-STEM
}

Frances I. Allen ${ }^{1,2^{*}}$, Thomas C. Pekin ${ }^{1,2^{* *}}$, Arun Persaud ${ }^{3}$, Steven J. Rozeveld ${ }^{4}$, Gregory F. Meyers ${ }^{4}$, Jim Ciston ${ }^{2}$, Colin Ophus ${ }^{2}$ and Andrew M. Minor ${ }^{1,2}$

1. Department of Materials Science and Engineering, UC Berkeley, Berkeley, CA, USA.

2. National Center for Electron Microscopy, Molecular Foundry, LBNL, Berkeley, CA, USA.

3. Accelerator Technology and Applied Physics Division, LBNL, Berkeley, CA, USA.

4. Core R\&D - Analytical Sciences, The Dow Chemical Company, Midland, MI, USA.

* Corresponding author: francesallen@berkeley.edu

** Current address: Department of Physics, Humboldt University of Berlin, Berlin, Germany.

Scanning nanobeam diffraction, propelled by the emergence of ultrafast direct-electron detectors, has become a popular technique in Scanning Transmission Electron Microscopy (STEM) for applications including nanoscale property mapping e.g. in situ strain mapping [1], and electron ptychography [2]. Since the electron probe is scanned over $2 \mathrm{D}$ co-ordinates $(\mathrm{x}, \mathrm{y})$ and a $2 \mathrm{D}$ diffraction with co-ordinates $\left(\mathrm{k}_{\mathrm{x}}, \mathrm{k}_{\mathrm{y}}\right)$ is acquired at each dwell point, the technique is also known as 4D-STEM. In the work presented here, we use 4D-STEM to achieve high-resolution, high-throughput grain orientation mapping of industrial catalyst nanoparticles. We use aberration-corrected STEM to obtain a sub-nanometer probe and a K2 directelectron detector to enable ultrafast data acquisition. Compared to Precession Electron Diffraction (PED), in which a set of diffraction patterns covering a range of orientations is collected for each point in the STEM scan, 4D-STEM is much faster and more straightforward to implement, albeit the level of indexing precision is inherently lower. In addition, by using ultrafast detection, we are able to efficiently map fields of view large enough to make robust statistical analysis of grain size distributions at sub-nanometer resolution possible.

4D-STEM datasets are intrinsically large and with ongoing detector development enabling image acquisition at ever increasing speeds, efficient data processing strategies are required. We investigate two multivariate statistical analysis techniques for the decomposition of our 4D-STEM datasets, Non-Negative Matrix Factorization (NNMF) and Principal Component Analysis (PCA). NNMF, while computationally significantly more time-intensive, has the benefit of generating output basis vectors that contain positive peaks only (a negative Bragg peak is in reality nonsensical) and thus the NNMF output is directly interpretable. In comparison, PCA computation is very fast, but the output basis vectors now include negative peaks, making a physical interpretation much more complex. Data pre-processing routines, including peak-finding algorithms to define the center points of all Bragg disks with sub-pixel precision, and reducing the disks to points in order to remove the potential influence of disk structure on the subsequent statistical analysis by NNMF/PCA, are also crucial [3].

Figure 1 shows the results of NNMF analysis of a subset of nanoparticles cropped from a larger dataset, demonstrating that grain classification with sub-nanometer resolution is achieved. The experiment was performed using the aberration-corrected TEAM 1 microscope at the National Center for Electron Microscopy, LBNL, operated at $300 \mathrm{kV}$ and equipped with a K2 direct electron detector. The electron beam was focused to a probe size of $0.5 \mathrm{~nm}$ (FWHM), the scan step size was set to $0.25 \mathrm{~nm}$ and the data acquired at 400 frames per second. Since PCA computation is much faster, a convenient strategy is to apply PCA first to obtain a scree plot from which the number of discrete grains in the dataset can be roughly estimated. This can then be used to guide the choice of the initial number of components to be run through NNMF. However, for the largest 4D-STEM datasets ( $>>1$ Gigabyte), relying on PCA alone may still be the only practical option depending on the computing resources available. [4] 


\section{References:}

[1] TC Pekin et al., Scripta Materialia 146 (2018), p. 87.

[2] Y Jiang et al., Nature 559 (2018), p. 343.

[3] TC Pekin et al., Ultramicroscopy 176 (2017), p. 170.

[4] The authors acknowledge support from the Dow University Partnership Initiative Program. Work at the Molecular Foundry was supported by the Office of Science, Office of Basic Energy Sciences, of the U.S. Department of Energy under Contract No. DE-AC02-05CH11231.
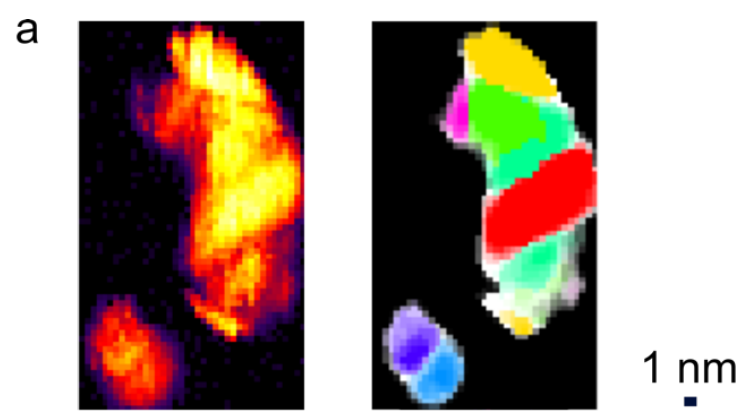

b

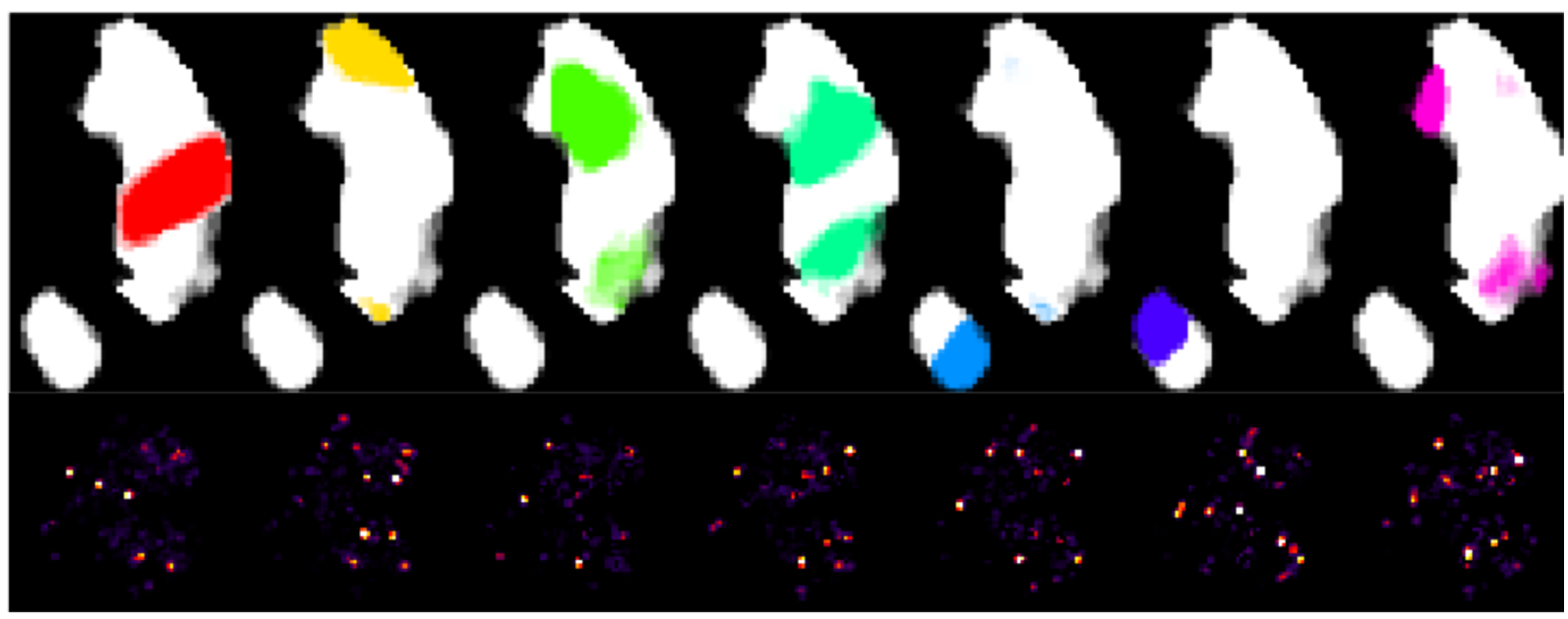

C

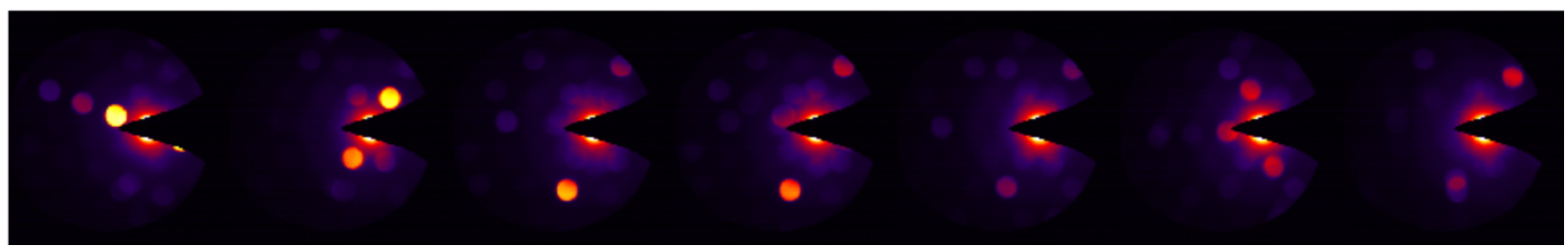

Figure 1. 4D-STEM grain mapping of gold-palladium nanoparticles. (a) Virtual dark-field STEM image of a nanoparticle cluster (pseudocolor, left), and composite grain map (right) obtained by NNMF. (b) Output basis vectors (top row) and corresponding score images (bottom row) identified by NNMF. (c) Diffraction patterns for each grain weighted using the respective score images. 